\title{
Efficient Estimator of a Finite Population Mean Using Two Auxiliary Variables and Numerical Application in Agricultural, Biomedical, and Power Engineering
}

\author{
Jingli Lu \\ College of Science, Inner Mongolia University of Technology, Hohhot, Inner Mongolia, China \\ Correspondence should be addressed to Jingli Lu; lujingli2004@163.com
}

Received 23 January 2017; Revised 21 July 2017; Accepted 25 July 2017; Published 23 August 2017

Academic Editor: Guido Ala

Copyright ( 2017 Jingli Lu. This is an open access article distributed under the Creative Commons Attribution License, which permits unrestricted use, distribution, and reproduction in any medium, provided the original work is properly cited.

To improve the efficiency of an estimator with two auxiliary variables, we propose a new estimator of a finite population mean under simple random sampling. The bias and mean square error expressions of the proposed estimator have been obtained. In a comparison study, we found that the new estimator was consistently better than those of Abu-Dayyeh et al., Kadilar and Cingi, and Malik and Singh, as well as the regression estimator using two auxiliary variables, and that the minimum MSE values of the previous three above reported estimators were equal. We used four numerical examples in agricultural, biomedical, and power engineering to support these theoretical results, thus enriching the theory of survey samples by the development of new estimators with two auxiliary variables.

\section{Introduction}

In sampling theory, it is a well-established phenomenon that supplementary information provided by auxiliary variables or auxiliary attributes often improves the accuracy of estimators of unknown population parameters. Ratio-, product-, and regression-type estimators are three such methods. For this reason, some authors have exploited the use of auxiliary variables and attributes at the estimation stage to increase estimator efficiency. For example, the planting area and the proportion of good seeds in agricultural engineering are two important auxiliary variables when estimating average cotton output. Similarly, the breed of cow in animal husbandry engineering is an important auxiliary attribute when estimating average milk yield. Thus, auxiliary information can be used in the field of education, biostatistics, the medical research, agricultural and biomedical engineering, and so on.

In the literature, some authors have proposed many efficient ratio-, product-, and regression-type estimators using one auxiliary variable or attribute, including Singh and Vishwakarma [1], Grover and Kaur [2, 3], Singh et al. [4], Singh and Solanki [5], and Gupta and Shabbir [6]. More recently, several authors have proposed efficient estimators of finite population mean using two variables or attributes, including, Abu-Dayyeh et al. [7], Kadilar and Cingi [8], Malik and Singh [9], Sharma and Singh [10], and Muneer et al. [11]. Although these studies are detailed and elaborated, the formulas of minimum MSE are not given, and the difference of minimum MSE values between these studies seems not to have been noticed.

In this paper, we compare the estimators reported by Abu-Dayyeh et al. [7], Kadilar and Cingi [8], and Malik and Singh [9] and introduce a new estimator with two auxiliary variables to estimate a finite population mean for the variable of interest. We obtained bias and mean square error (MSE) equations for the proposed estimator, and we compared the new estimator against those with relatively high efficiencies. An empirical study using four datasets in agricultural, biomedical, and power engineering was conducted, and we obtained satisfactory results, both theoretically and numerically. The analysis of these issues is of great significance for understanding agricultural, biomedical, and power engineering. Therefore, the proposed estimator could be applied across a broad spectrum of sampling survey. 


\section{Materials and Methods}

2.1. Abu-Dayyeh Estimator. Abu-Dayyeh et al. [7] proposed the following estimator of population mean when the population means $\bar{X}_{1}$ and $\bar{X}_{2}$ of the auxiliary variables were known:

$$
\bar{y}_{\mathrm{AD}}=\bar{y}\left(\frac{\bar{X}_{1}}{\bar{x}_{1}}\right)^{\alpha_{1}}\left(\frac{\bar{X}_{2}}{\bar{x}_{2}}\right)^{\alpha_{2}},
$$

where $\bar{y}$ denotes the sample means of the variable $y, \bar{x}_{i}$ and $\bar{X}_{i}$ $(i=1,2)$ denote, respectively, the sample and the population means of the variable $x_{i}(i=1,2)$, and $\alpha_{1}$ and $\alpha_{2}$ are real numbers.

The MSE of $\bar{y}_{\mathrm{AD}}$ is given by

$$
\begin{aligned}
& \operatorname{MSE}\left(\bar{y}_{\mathrm{AD}}\right) \cong \frac{1-f}{n} \bar{Y}^{2}\left(C_{y}^{2}+\alpha_{1}^{2} C_{x_{1}}^{2}+\alpha_{2}^{2} C_{x_{2}}^{2}\right. \\
& \quad-2 \alpha_{1} C_{y} C_{x_{1}} \rho_{y x_{1}}-2 \alpha_{2} C_{y} C_{x_{2}} \rho_{y x_{2}} \\
& \left.\quad+2 \alpha_{1} \alpha_{2} C_{x_{1}} C_{x_{2}} \rho_{x_{1} x_{2}}\right),
\end{aligned}
$$

where $f=n / N ; n$ and $N$ are, respectively, the number of units in the sample and the population; $C_{y}^{2}, C_{x_{1}}^{2}$, and $C_{x_{2}}^{2}$ are the coefficients of variation of $Y, X_{1}$, and $X_{2}$, respectively; and $\rho_{x_{1} x_{2}}, \rho_{y x_{1}}$, and $\rho_{y x_{2}}$ are the correlation coefficients between $X_{1}$ and $X_{2}, Y$ and $X_{1}$, and $Y$ and $X_{2}$, respectively.

To minimize $\operatorname{MSE}\left(\bar{y}_{\mathrm{AD}}\right)$, the optimum values of $\alpha_{1}$ and $\alpha_{2}$ are given by

$$
\begin{aligned}
& \alpha_{1}^{*}=\frac{C_{y}\left(\rho_{y x_{1}}-\rho_{x_{1} x_{2}} \rho_{y x_{2}}\right)}{C_{x_{1}}\left(1-\rho_{x_{1} x_{2}}^{2}\right)}, \\
& \alpha_{2}^{*}=\frac{C_{y}\left(\rho_{y x_{2}}-\rho_{x_{1} x_{2}} \rho_{y x_{1}}\right)}{C_{x_{2}}\left(1-\rho_{x_{1} x_{2}}^{2}\right)} .
\end{aligned}
$$

The minimum MSE of $\bar{y}_{\mathrm{AD}}$ can be shown as

$$
\operatorname{MSE}_{\text {min }}\left(\bar{y}_{\mathrm{AD}}\right)=\frac{\delta \bar{Y}^{2} C_{y}^{2} L}{A} \text {, }
$$

where $\delta=(1-f) / n ; L=1-\rho_{x_{1} x_{2}}^{2}-\rho_{y x_{1}}^{2}+2 \rho_{x_{1} x_{2}} \rho_{y x_{1}} \rho_{y x_{2}}-\rho_{y x_{2}}^{2}$; $A=1-\rho_{x_{1} x_{2}}^{2}$.

2.2. Kadilar and Cingi Estimator. Kadilar and Cingi [8] proposed an estimator using two auxiliary variables, $x_{1}$ and $x_{2}$, to estimate the population mean $\bar{Y}$, as follows:

$$
\begin{aligned}
\bar{y}_{\mathrm{KC}}= & \bar{y}\left(\frac{\bar{X}_{1}}{\bar{x}_{1}}\right)^{\alpha_{1}}\left(\frac{\bar{X}_{2}}{\bar{x}_{2}}\right)^{\alpha_{2}}+b_{1}\left(\bar{X}_{1}-\bar{x}_{1}\right) \\
& +b_{2}\left(\bar{X}_{2}-\bar{x}_{2}\right),
\end{aligned}
$$

where $b_{1}=S_{y x_{1}} / S_{x_{1}}^{2}$ and $b_{2}=S_{y x_{2}} / S_{x_{2}}^{2} ; S_{x_{1}}^{2}$ and $S_{x_{2}}^{2}$ are the variances of $Y, X_{1}$, and $X_{2}$, respectively; and $S_{y x_{1}}$ and $S_{y x_{2}}$ are the covariance between $Y$ and $X_{1}$ and $Y$ and $X_{2}$, respectively.
The MSE of $\bar{y}_{\mathrm{KC}}$ is given by

$$
\begin{aligned}
& \operatorname{MSE}\left(\bar{y}_{\mathrm{KC}}\right) \cong \frac{1-f}{n} \bar{Y}^{2}\left(C_{y}^{2}+\alpha_{1}^{2} C_{x_{1}}^{2}+\alpha_{2}^{2} C_{x_{2}}^{2}\right. \\
& -2 \alpha_{1} C_{y} C_{x_{1}} \rho_{x_{1} x_{2}} \rho_{y x_{2}}-2 \alpha_{2} C_{y} C_{x_{2}} \rho_{x_{1} x_{2}} \rho_{y x_{1}} \\
& +2 \alpha_{1} \alpha_{2} C_{x_{1}} C_{x_{2}} \rho_{x_{1} x_{2}}-C_{y}^{2} \rho_{y x_{1}}^{2}-C_{y}^{2} \rho_{y x_{2}}^{2} \\
& \left.+2 C_{y}^{2} \rho_{x_{1} x_{2}} \rho_{y x_{1}} \rho_{y x_{2}}\right) .
\end{aligned}
$$

To minimize $\operatorname{MSE}\left(\bar{y}_{\mathrm{KC}}\right)$, the optimum values of $\alpha_{1}$ and $\alpha_{2}$ are given by

$$
\begin{aligned}
& \alpha_{1}^{*}=\frac{C_{y} \rho_{x_{1} x_{2}}\left(\rho_{y x_{1}} \rho_{x_{1} x_{2}}-\rho_{y x_{2}}\right)}{C_{x_{1}}\left(1-\rho_{x_{1} x_{2}}^{2}\right)}, \\
& \alpha_{2}^{*}=\frac{C_{y} \rho_{x_{1} x_{2}}\left(\rho_{y x_{2}} \rho_{x_{1} x_{2}}-\rho_{y x_{1}}\right)}{C_{x_{2}}\left(1-\rho_{x_{1} x_{2}}^{2}\right)} .
\end{aligned}
$$

The minimum MSE of $\bar{y}_{\mathrm{KC}}$ can be shown as

$$
\operatorname{MSE}_{\min }\left(\bar{y}_{\mathrm{KC}}\right)=\frac{\delta \bar{Y}^{2} C_{y}^{2} L}{A} .
$$

2.3. Malik and Singh Estimator. Malik and Singh [9] proposed an estimator to estimate the population mean $\bar{Y}$, as follows:

$$
\begin{aligned}
\bar{y}_{\mathrm{MS}}= & \bar{y} \exp \left(\frac{\bar{X}_{1}-\bar{x}_{1}}{\bar{X}_{1}+\bar{x}_{1}}\right)^{\beta_{1}}\left(\frac{\bar{X}_{2}-\bar{x}_{2}}{\bar{X}_{2}+\bar{x}_{2}}\right)^{\beta_{2}} \\
& +b_{1}\left(\bar{X}_{1}-\bar{x}_{1}\right)+b_{2}\left(\bar{X}_{2}-\bar{x}_{2}\right),
\end{aligned}
$$

where $\beta_{1}$ and $\beta_{2}$ are real numbers.

The MSE of $\bar{y}_{\mathrm{MS}}$ is given by

$$
\begin{aligned}
& \operatorname{MSE}\left(\bar{y}_{\mathrm{MS}}\right) \cong \delta\left[\overline { Y } ^ { 2 } \left(C_{y}^{2}+\frac{1}{4} \beta_{1}^{2} C_{x_{1}}^{2}+\frac{1}{4} \beta_{2}^{2} C_{x_{2}}^{2}\right.\right. \\
& +\frac{1}{2} \beta_{1} \beta_{2} C_{x_{1}} C_{x_{2}} \rho_{x_{1} x_{2}}-\beta_{1} C_{x_{1}} C_{y} \rho_{y x_{1}} \\
& \left.\quad-\beta_{2} C_{x_{2}} C_{y} \rho_{y x_{2}}\right)+\bar{Y}\left(\beta_{1} b_{1} C_{x_{1}}^{2} \bar{X}_{1}+\beta_{2} b_{2} C_{x_{2}}^{2} \bar{X}_{2}\right. \\
& +\beta_{1} b_{2} C_{x_{1}} C_{x_{2}} \rho_{x_{1} x_{2}} \bar{X}_{2}+\beta_{2} b_{1} C_{x_{1}} C_{x_{2}} \rho_{x_{1} x_{2}} \bar{X}_{1} \\
& \left.-2 b_{1} C_{x_{1}} C_{y} \rho_{y x_{1}} \bar{X}_{1}-2 b_{2} C_{x_{2}} C_{y} \rho_{y x_{2}} \bar{X}_{2}\right)+b_{1}^{2} C_{x_{1}}^{2} \bar{X}_{1}^{2} \\
& \left.+2 b_{1} b_{2} C_{x_{1}} C_{x_{2}} \rho_{x_{1} x_{2}} \bar{X}_{1} \bar{X}_{2}+b_{2}^{2} C_{x_{2}}^{2} \bar{X}_{2}^{2}\right] .
\end{aligned}
$$

To minimize $\operatorname{MSE}\left(\bar{y}_{\mathrm{MS}}\right)$, the optimum values of $\beta_{1}$ and $\beta_{2}$ are given by

$$
\begin{aligned}
& \beta_{1}^{*}=\frac{2 C_{y} \rho_{x_{1} x_{2}}\left(\rho_{y x_{1}} \rho_{x_{1} x_{2}}-\rho_{y x_{2}}\right)}{C_{x_{1}}\left(1-\rho_{x_{1} x_{2}}^{2}\right)}, \\
& \beta_{2}^{*}=\frac{C_{y} \rho_{x_{1} x_{2}}\left(\rho_{y x_{2}} \rho_{x_{1} x_{2}}-\rho_{y x_{1}}\right)}{C_{x_{2}}\left(1-\rho_{x_{1} x_{2}}\right)} .
\end{aligned}
$$


The minimum MSE of $\bar{y}_{\mathrm{KC}}$ can be shown as

$$
\operatorname{MSE}_{\text {min }}\left(\bar{y}_{\mathrm{MS}}\right)=\frac{\delta \bar{Y}^{2} C_{y}^{2} L}{A} .
$$

2.4. The Regression Estimator. Rao [12] proposed an estimator using one auxiliary variable, $x_{1}$, to estimate the population mean $\bar{Y}$, as follows:

$$
\bar{y}_{\text {Rao }}=w_{1} \bar{y}+w_{2}\left(\bar{X}_{1}-\bar{x}_{1}\right) .
$$

Similarly, following Rao, a regression estimator of $\bar{Y}$ using two auxiliary variables, $x_{1}$ and $x_{2}$, is given by

$$
\bar{y}_{\mathrm{RE}}=w_{1} \bar{y}+w_{2}\left(\bar{X}_{1}-\bar{x}_{1}\right)+w_{3}\left(\bar{X}_{2}-\bar{x}_{2}\right),
$$

where $w_{1}, w_{2}$, and $w_{3}$ are real constants.

The MSE of $\bar{y}_{\mathrm{RE}}$ is given by

$$
\begin{aligned}
& \operatorname{MSE}\left(\bar{y}_{\mathrm{RE}}\right)=\bar{Y}^{2}+w_{1}^{2}\left(1+\delta C_{y}^{2}\right)+\delta\left\{\bar{X}_{2}^{2} C_{x_{2}}^{2} w_{3}^{2}\right. \\
& \left.\quad+\bar{X}_{1} C_{x_{1}} w_{2}\left(\bar{X}_{1} C_{x_{1}} w_{2}+2 \bar{X}_{2} C_{x_{2}} w_{3} \rho_{x_{1} x_{2}}\right)\right\} \\
& \quad-2 \bar{Y} w_{1}\{\bar{Y} \\
& \left.\quad+\delta C_{y}\left(\bar{X}_{1} C_{x_{1}} w_{2} \rho_{y x_{1}}+\bar{X}_{2} C_{x_{2}} w_{3} \rho_{y x_{2}}\right)\right\} .
\end{aligned}
$$

The optimum values of $w_{1}, w_{2}$, and $w_{3}$, obtained by minimizing (15), respectively, are given by

$$
\begin{aligned}
& w_{1}^{*}=\frac{A}{A+\delta C_{y}^{2} L}, \\
& w_{2}^{*}=\frac{\bar{Y} C_{y}\left(\rho_{y x_{1}}-\rho_{x_{1} x_{2}} \rho_{y x_{2}}\right)}{C_{x_{1}} \bar{X}_{1}\left(A+\delta C_{y}^{2} L\right)}, \\
& w_{3}^{*}=\frac{\bar{Y} C_{y}\left(\rho_{y x_{2}}-\rho_{x_{1} x_{2}} \rho_{y x_{1}}\right)}{C_{x_{2}} \bar{X}_{2}\left(A+\delta C_{y}^{2} L\right)} .
\end{aligned}
$$

The minimum MSE of $\bar{y}_{\mathrm{RE}}$ can be shown as

$$
\operatorname{MSE}_{\text {min }}\left(\bar{y}_{\mathrm{RE}}\right)=\frac{\delta \bar{Y}^{2} C_{y}^{2} L}{A+\delta C_{y}^{2} L} \text {. }
$$

2.5. The Proposed Estimator. Singh and Espejo [13] proposed an estimator using one auxiliary variable, $x$, to estimate the population mean $\bar{Y}$, as follows:

$$
\bar{Y}_{\mathrm{SE}}=\frac{\bar{y}}{2}\left(\frac{\bar{X}}{\bar{x}}+\frac{\bar{x}}{\bar{X}}\right)
$$

Inspired by this work, we propose a new estimator with two auxiliary variables, as follows:

$$
\begin{aligned}
\bar{y}_{\mathrm{pr}} & =\frac{k_{1} \bar{y}+k_{2}\left(\bar{X}_{1}-\bar{x}_{1}\right)+k_{3}\left(\bar{X}_{2}-\bar{x}_{2}\right)}{4}\left(\frac{\bar{X}_{1}}{\bar{x}_{1}}\right. \\
& \left.+\frac{\bar{x}_{1}}{\bar{X}_{1}}\right)\left(\frac{\bar{X}_{2}}{\bar{x}_{2}}+\frac{\bar{x}_{2}}{\bar{X}_{2}}\right),
\end{aligned}
$$

where $k_{1}, k_{2}$, and $k_{3}$ are real constants.
Let $\varepsilon_{0}=\bar{y} / \bar{Y}-1, \varepsilon_{1}=\bar{x}_{1} / \bar{X}_{1}-1$, and $\varepsilon_{2}=\bar{x}_{2} / \bar{X}_{2}-1$. Under simple random sampling without replacement (SRSWOR), we have the following expectations:

$$
\begin{aligned}
E\left(\varepsilon_{0}\right) & =E\left(\varepsilon_{1}\right)=E\left(\varepsilon_{2}\right)=0, \\
E\left(\varepsilon_{0}^{2}\right) & =\delta C_{y}^{2}, \\
E\left(\varepsilon_{1}^{2}\right) & =\delta C_{x_{1}}^{2}, \\
E\left(\varepsilon_{2}^{2}\right) & =\delta C_{x_{2}}^{2}, \\
E\left(\varepsilon_{0} \varepsilon_{1}\right) & =\delta \rho_{y x_{1}} C_{y} C_{x_{1}}, \\
E\left(\varepsilon_{0} \varepsilon_{2}\right) & =\delta \rho_{y x_{2}} C_{y} C_{x_{2}}, \\
E\left(\varepsilon_{1} \varepsilon_{2}\right) & =\delta \rho_{x_{1} x_{2}} C_{x_{1}} C_{x_{2}} .
\end{aligned}
$$

The proposed estimator $\bar{y}_{\mathrm{pr}}$ can be rewritten as

$$
\begin{aligned}
\bar{y}_{\mathrm{pr}} & =\frac{k_{1} \bar{Y}\left(\varepsilon_{0}+1\right)-k_{2} \bar{X}_{1} \varepsilon_{1}-k_{3} \bar{X}_{2} \varepsilon_{2}}{4}\left(\frac{1}{\varepsilon_{1}+1}+\varepsilon_{1}\right. \\
& +1)\left(\frac{1}{\varepsilon_{2}+1}+\varepsilon_{2}+1\right) .
\end{aligned}
$$

By rewriting $\bar{y}_{\mathrm{pr}}$, we have

$$
\begin{aligned}
\bar{y}_{\mathrm{pr}} & =\frac{k_{1} \bar{Y}\left(\varepsilon_{0}+1\right)-k_{2} \bar{X}_{1} \varepsilon_{1}-k_{3} \bar{X}_{2} \varepsilon_{2}}{4}\left[\varepsilon_{1}+1\right. \\
& \left.+\left(1-\varepsilon_{1}+\varepsilon_{1}^{2}+\cdots\right)\right]\left[\varepsilon_{2}+1\right. \\
& \left.+\left(1-\varepsilon_{2}+\varepsilon_{2}^{2}+\cdots\right)\right] .
\end{aligned}
$$

By retaining only the terms up to the second degree of $\varepsilon$ 's, we have

$$
\begin{aligned}
\bar{y}_{\mathrm{pr}}-\bar{Y} \cong & \left(k_{1}-1\right) \bar{Y}+k_{1} \bar{Y} \varepsilon_{0}-k_{2} \bar{X}_{1} \varepsilon_{1}-k_{3} \bar{X}_{2} \varepsilon_{2} \\
& +\frac{1}{2} k_{1} \bar{Y} \varepsilon_{1}^{2}+\frac{1}{2} k_{1} \bar{Y} \varepsilon_{2}^{2} .
\end{aligned}
$$

The bias of the proposed estimator is given by

$$
\begin{aligned}
\operatorname{Bias}\left(\bar{y}_{\mathrm{pr}}\right) & =E\left(\bar{y}_{\mathrm{pr}}\right)-\bar{Y} \\
& \cong \bar{Y}\left[\left(k_{1}-1\right)+\frac{1}{2} \delta k_{1} C_{x_{1}}^{2}+\frac{1}{2} \delta k_{1} C_{x_{2}}^{2}\right] .
\end{aligned}
$$

The MSE of this new estimator with two auxiliary variables is given by

$$
\begin{aligned}
& \operatorname{MSE}\left(\bar{y}_{\mathrm{pr}}\right)=E\left(\bar{y}_{\mathrm{pr}}-\bar{Y}\right)^{2} \cong \bar{Y}^{2}\left[\left(k_{1}-1\right)^{2}\right. \\
& \left.-\delta k_{1}\left(C_{x_{1}}^{2}+C_{x_{2}}^{2}\right)+\delta k_{1}^{2}\left(C_{x_{1}}^{2}+C_{x_{2}}^{2}+C_{y}^{2}\right)\right] \\
& -2 \delta k_{1} \bar{Y} C_{y}\left(k_{2} C_{x_{1}} \rho_{y x_{1}} \bar{X}_{1}+k_{3} C_{x_{2}} \rho_{y x_{2}} \bar{X}_{2}\right) \\
& +\delta\left(k_{2}^{2} C_{x_{1}}^{2} \bar{X}_{1}^{2}+2 C_{x_{1}} C_{x_{2}} k_{2} k_{3} \rho_{x_{1} x_{2}} \bar{X}_{1} \bar{X}_{2}\right. \\
& \left.+k_{3}^{2} C_{x_{2}}^{2} \bar{X}_{2}^{2}\right) .
\end{aligned}
$$


The optimum values of $k_{1}, k_{2}$, and $k_{3}$ are given by

$$
\begin{aligned}
& k_{1}^{*}=\frac{A\left[2+\delta\left(C_{x_{1}}^{2}+C_{x_{2}}^{2}\right)\right]}{2\left(A+C_{y}^{2} \delta L+A \delta\left(C_{x_{1}}^{2}+C_{x_{2}}^{2}\right)\right)}, \\
& k_{2}^{*}=\frac{C_{y} \bar{Y}\left(\rho_{y x_{1}}-\rho_{x_{1} x_{2}} \rho_{y x_{2}}\right)\left[2+\delta\left(C_{x_{1}}^{2}+C_{x_{2}}^{2}\right)\right]}{2 C_{x_{1}} \bar{X}_{1}\left(A+C_{y}^{2} \delta L+A \delta\left(C_{x_{1}}^{2}+C_{x_{2}}^{2}\right)\right)}, \\
& k_{3}^{*}=\frac{C_{y} \bar{Y}\left(\rho_{y x_{2}}-\rho_{x_{1} x_{2}} \rho_{y x_{1}}\right)\left[2+\delta\left(C_{x_{1}}^{2}+C_{x_{2}}^{2}\right)\right]}{2 C_{x_{2}} \bar{X}_{2}\left(A+C_{y}^{2} \delta L+A \delta\left(C_{x_{1}}^{2}+C_{x_{2}}^{2}\right)\right)} .
\end{aligned}
$$

The minimum MSE of $\bar{y}_{\mathrm{pr}}$ can be shown as

$$
\operatorname{MSE}_{\min }\left(\bar{y}_{\mathrm{pr}}\right)=\frac{\delta \bar{Y}^{2}\left[4 C_{y}^{2} L-A \delta\left(C_{x_{1}}^{2}+C_{x_{2}}^{2}\right)^{2}\right]}{4\left[A+C_{y}^{2} \delta L+A \delta\left(C_{x_{1}}^{2}+C_{x_{2}}^{2}\right)\right]}
$$

2.6. Comparison of $\bar{y}_{p r}$ with Some Existing Estimators. We compared the MSE of the proposed estimator with two auxiliary variables given in (27) with the MSE of the estimator reported by Abu-Dayyeh et al. [7], as given in (4), Kadilar and Cingi [8], as given in (8), Malik and Singh [9], as given in (12), and the regression estimator, as given in (17), as follows:

$$
\begin{aligned}
\operatorname{MSE}_{\min }\left(\bar{y}_{\mathrm{pr}}\right) & <\operatorname{MSE}_{\text {min }}\left(\bar{y}_{\mathrm{RE}}\right)<\operatorname{MSE}_{\min }\left(\bar{y}_{\mathrm{AD}}\right) \\
& =\operatorname{MSE}_{\min }\left(\bar{y}_{\mathrm{KC}}\right)=\operatorname{MSE}_{\min }\left(\bar{y}_{\mathrm{MS}}\right),
\end{aligned}
$$

always.

Proof.

$$
\begin{aligned}
& \operatorname{MSE}_{\min }\left(\bar{y}_{\mathrm{RE}}\right)=\frac{\delta \bar{Y}^{2} C_{y}^{2} L}{A+\delta C_{y}^{2} L}=\frac{\delta \bar{Y}^{2} C_{y}^{2} L / A}{1+\left(\delta \bar{Y}^{2} C_{y}^{2} L / A\right)\left(1 / \bar{Y}^{2}\right)} \\
& =\frac{\operatorname{MSE}_{\min }\left(\bar{y}_{\mathrm{AD}}\right)}{1+\operatorname{MSE}_{\min }\left(\bar{y}_{\mathrm{AD}}\right) / \bar{Y}^{2}}<\mathrm{MSE}_{\min }\left(\bar{y}_{\mathrm{AD}}\right) \\
& =\operatorname{MSE}_{\min }\left(\bar{y}_{\mathrm{KC}}\right)=\mathrm{MSE}_{\min }\left(\bar{y}_{\mathrm{MS}}\right), \\
& \operatorname{MSE}_{\min }\left(\bar{y}_{\mathrm{pr}}\right)=\frac{\delta \bar{Y}^{2}\left[4 C_{y}^{2} L-A \delta\left(C_{x_{1}}^{2}+C_{x_{2}}^{2}\right)^{2}\right]}{4\left[A+C_{y}^{2} \delta L+A \delta\left(C_{x_{1}}^{2}+C_{x_{2}}^{2}\right)\right]} \\
& =\frac{\delta \bar{Y}^{2} C_{y}^{2} L}{\left[A+C_{y}^{2} \delta L+A \delta\left(C_{x_{1}}^{2}+C_{x_{2}}^{2}\right)\right]} \\
& -\frac{\delta^{2} \bar{Y}^{2} A\left(C_{x_{1}}^{2}+C_{x_{2}}^{2}\right)^{2}}{4\left[A+C_{y}^{2} \delta L+A \delta\left(C_{x_{1}}^{2}+C_{x_{2}}^{2}\right)\right]}
\end{aligned}
$$

$$
\begin{aligned}
& =\frac{\delta \bar{Y}^{2} C_{y}^{2} L /\left(A+C_{y}^{2} \delta L\right)}{\left[1+A \delta\left(C_{x_{1}}^{2}+C_{x_{2}}^{2}\right) /\left(A+C_{y}^{2} \delta L\right)\right]} \\
& -\frac{\delta^{2} \bar{Y}^{2} A\left(C_{x_{1}}^{2}+C_{x_{2}}^{2}\right)^{2} /\left(A+C_{y}^{2} \delta L\right)}{4\left[1+A \delta\left(C_{x_{1}}^{2}+C_{x_{2}}^{2}\right) /\left(A+C_{y}^{2} \delta L\right)\right]} \\
& =\frac{\mathrm{MSE}_{\min }\left(\bar{y}_{\mathrm{RE}}\right)}{\left[1+\delta\left(C_{x_{1}}^{2}+C_{x_{2}}^{2}\right) \mathrm{MSE}_{\min }\left(\bar{y}_{\mathrm{RE}}\right) / \mathrm{MSE}_{\min }\left(\bar{y}_{\mathrm{KC}}\right)\right]} \\
& -\frac{\delta^{2} \bar{Y}^{2}\left(C_{x_{1}}^{2}+C_{x_{2}}^{2}\right)^{2} \mathrm{MSE}_{\min }\left(\bar{y}_{\mathrm{RE}}\right) / \mathrm{MSE}_{\min }\left(\bar{y}_{\mathrm{KC}}\right)}{4\left[1+\delta\left(C_{x_{1}}^{2}+C_{x_{2}}^{2}\right) \mathrm{MSE}_{\min }\left(\bar{y}_{\mathrm{RE}}\right) / \mathrm{MSE}_{\min }\left(\bar{y}_{\mathrm{KC}}\right)\right]} \\
& =\frac{\mathrm{MSE}_{\min }\left(\bar{y}_{\mathrm{RE}}\right)}{1+M}-\frac{\bar{Y}^{2} \delta\left(C_{x_{1}}^{2}+C_{x_{2}}^{2}\right) M}{4(1+M)},
\end{aligned}
$$

where $M=\delta\left(C_{x_{1}}^{2}+C_{x_{2}}^{2}\right) \operatorname{MSE}_{\min }\left(\bar{y}_{\mathrm{RE}}\right) / \operatorname{MSE}_{\min }\left(\bar{y}_{\mathrm{KC}}\right)$

$$
\begin{aligned}
& M=\frac{\delta\left(C_{x_{1}}^{2}+C_{x_{2}}^{2}\right) \mathrm{MSE}_{\min }\left(\bar{y}_{\mathrm{RE}}\right)}{\mathrm{MSE}_{\min }\left(\bar{y}_{\mathrm{KC}}\right)}>0 \Longrightarrow \\
& \frac{\bar{Y}^{2} \delta\left(C_{x_{1}}^{2}+C_{x_{2}}^{2}\right) M}{4(1+M)}>0 \Longrightarrow \\
& \operatorname{MSE}_{\min }\left(\bar{y}_{\mathrm{pr}}\right)<\frac{\mathrm{MSE}_{\min }\left(\bar{y}_{\mathrm{RE}}\right)}{1+M}<\mathrm{MSE}_{\min }\left(\bar{y}_{\mathrm{RE}}\right) .
\end{aligned}
$$

2.7. Numerical Application in Engineering. To examine the merits of the proposed estimator, we considered four natural population datasets in agricultural, biomedical, and power engineering. We used the following formula to calculate the percent of relative efficiency of different estimators:

$$
\operatorname{PRE}\left(\phi, \bar{y}_{\mathrm{AD}}\right)=\frac{\operatorname{MSE}\left(\bar{y}_{\mathrm{AD}}\right)}{\operatorname{MSE}(\phi)} \times 100,
$$

where $\phi=\bar{y}_{\mathrm{RE}}$ or $\bar{y}_{\mathrm{KC}}$ or $\bar{y}_{\mathrm{MS}}$ or $\bar{y}_{\mathrm{pr}}$.

Population I (source in biomedical engineering [14])

$Y$ : number of "placebo" children.

$X_{1}$ : number of paralytic polio cases in the placebo group.

$X_{2}$ : number of paralytic polio cases in the "not inoculated" group.

$N=34, \bar{Y}=4.92, \bar{X}_{1}=2.59, \bar{X}_{2}=2.91, \rho_{y x_{1}}=$ $0.7326, \rho_{y x_{2}}=0.6430, \rho_{x_{1} x_{2}}=0.6837, C_{y}=1.0123$, $C_{x_{1}}=1.2319$, and $C_{x_{2}}=1.0720$.

Population II (source in agricultural engineering)

$Y$ : cotton output.

$X_{1}$ : the area of the plant. 
TABLE 1: MSE and PRE values of different estimators about population I.

\begin{tabular}{lccccccc}
\hline & $n$ & $n / N$ & $\bar{y}_{\mathrm{AD}}$ & $\bar{y}_{\mathrm{KC}}$ & $\bar{y}_{\mathrm{MS}}$ & $\bar{y}_{\mathrm{RE}}$ & $\bar{y}_{\mathrm{pr}}$ \\
\hline \multirow{3}{*}{$\mathrm{MSE}$} & 10 & 0.294 & 0.738607 & 0.738607 & 0.738607 & 0.716737 & 0.433563 \\
& 15 & 0.441 & 0.390406 & 0.390406 & 0.390406 & 0.384209 & 0.297354 \\
& 20 & 0.588 & 0.211030 & 0.211030 & 0.211030 & 0.209207 & 0.182492 \\
& 25 & 0.735 & 0.105515 & 0.105515 & 0.105515 & 0.105057 & 0.098166 \\
\hline \multirow{3}{*}{$\operatorname{PRE}\left(\phi, \bar{y}_{\mathrm{AD}}\right)$} & 10 & 0.294 & 100 & 100 & 100 & 103.05 & 170.36 \\
& 15 & 0.441 & 100 & 100 & 100 & 1.61 \\
& 20 & 0.588 & 100 & 100 & 100 & 100.87 \\
\hline
\end{tabular}

TABLE 2: MSE and PRE values of different estimators about population II.

\begin{tabular}{|c|c|c|c|c|c|c|c|}
\hline & $n$ & $n / N$ & $\bar{y}_{\mathrm{AD}}$ & $\bar{y}_{\mathrm{KC}}$ & $\bar{y}_{\mathrm{MS}}$ & $\bar{y}_{\mathrm{RE}}$ & $\bar{y}_{\mathrm{pr}}$ \\
\hline \multirow{4}{*}{ MSE } & 10 & 0.056 & 1.703302 & 1.703302 & 1.703302 & 1.688617 & 1.607564 \\
\hline & 50 & 0.278 & 0.260505 & 0.260505 & 0.260505 & 0.260159 & 0.258197 \\
\hline & 90 & 0.500 & 0.100194 & 0.100194 & 0.100194 & 0.100143 & 0.099852 \\
\hline & 130 & 0.722 & 0.038536 & 0.038536 & 0.038536 & 0.038529 & 0.038486 \\
\hline \multirow{4}{*}{$\operatorname{PRE}\left(\phi, \bar{y}_{\mathrm{AD}}\right)$} & 10 & 0.056 & 100 & 100 & 100 & 100.870 & 105.955 \\
\hline & 50 & 0.278 & 100 & 100 & 100 & 101.133 & 100.894 \\
\hline & 90 & 0.500 & 100 & 100 & 100 & 100.051 & 100.343 \\
\hline & 130 & 0.722 & 100 & 100 & 100 & 100.020 & 100.132 \\
\hline
\end{tabular}

$X_{2}$ : the proportion of good seed.

$N=180, \bar{Y}=13.9951, \bar{X}_{1}=27.3981, \bar{X}_{2}=38.7167$,

$\rho_{y x_{1}}=0.5630, \rho_{y x_{2}}=0.5273, \rho_{x_{1} x_{2}}=0.2589, C_{y}=$

$0.4180, C_{x_{1}}=0.4254$, and $C_{x_{2}}=0.3339$.

Population III (source in biomedical engineering [14])

$Y$ : weight measurement of children.

$X_{1}$ : midarm circumference of children.

$X_{2}$ : skull circumference of children.

$N=55, \bar{Y}=17.08, \bar{X}_{1}=16.92, \bar{X}_{2}=50.44, \rho_{y x_{1}}=$

$0.54, \rho_{y x_{2}}=0.51, \rho_{x_{1} x_{2}}=-0.08, C_{y}=0.1269, C_{x_{1}}=$ $0.07, C_{x_{2}}=0.0265$.

Population IV (source in power engineering)

$Y$ : Electricity consumption by region in China in 2002.

$X_{1}$ : Electricity consumption by region in China in 2001.

$X_{2}$ : Electricity consumption by region in China in 2000 .

$N=30, \bar{Y}=546.09, \bar{X}_{1}=489.29, \bar{X}_{2}=453.54$,

$\rho_{y x_{1}}=0.9986, \rho_{y x_{2}}=0.9931, \rho_{x_{1} x_{2}}=0.9965, C_{y}=$ $0.6942, C_{x_{1}}=0.6719$, and $C_{x_{2}}=0.6529$.

\section{Results and Discussion}

MSE and PRE values of different estimators about population I can be seen in Table 1.
MSE and PRE values of different estimators about population II can be seen in Table 2 .

MSE and PRE values of different estimators about population III can be seen in Table 3 .

MSE and PRE values of different estimators about population IV can be seen in Table 4.

The relative efficiency was studied based on the traditional regression- or ratio-type estimators in many literatures (Abu-Dayyeh et al. [7], Haq and Shabbir [15], and Verma et al. [16]). However, we studied the relative efficiency based on the higher efficient estimators using two auxiliary variables and found that the efficiency of the proposed estimator is higher than the estimators noted above under any conditions.

Under different sample sizes and different datasets, we notice from the data given in Tables 1, 2, 3, and 4 that the proposed estimator of a finite population mean using two auxiliary variables is always more efficient than the estimators $\bar{y}_{\mathrm{AD}}, \bar{y}_{\mathrm{KC}}, \bar{y}_{\mathrm{MS}}$, and $\bar{y}_{\mathrm{RE}}$. Although the expressions of the estimators reported by Abu-Dayyeh et al., Kadilar and Cingi, and Malik and Singh are different, we note from the comparative study above that the minimum MSE values for the estimators reported by Abu-Dayyeh et al. [7], Kadilar and Cingi [8], and Malik and Singh [9] are equal and have same expression. The regression estimator has a smaller MSE value than the three estimators noted above. With increase in sample fraction, the MSE and PRE values of the proposed estimator decrease. Therefore, a smaller sampling fraction yields better results relative to MSE and PRE values when compared to a larger sampling fraction. Moreover, with a large sampling fraction, the efficiency differential among all estimators in the present study is very small. Therefore, it is suggested that our estimators be used with small sampling 
TABLE 3: MSE and PRE values of different estimators about population III.

\begin{tabular}{lccccccc}
\hline & $n$ & $n / N$ & $\bar{y}_{\mathrm{AD}}$ & $\bar{y}_{\mathrm{KC}}$ & $\bar{y}_{\mathrm{MS}}$ & $\bar{y}_{\mathrm{RE}}$ & $\bar{y}_{\mathrm{pr}}$ \\
\hline \multirow{3}{*}{$\mathrm{MSE}$} & 10 & 0.182 & 0.15048093 & 0.15048093 & 0.15048093 & 0.15040335 & 0.15032137 \\
& 20 & 0.364 & 0.05981617 & 0.05981617 & 0.05981617 & 0.05980391 & 0.05979094 \\
& 30 & 0.545 & 0.02821517 & 0.02821517 & 0.02821517 & 0.02821245 & 0.0282096 \\
& 40 & 0.727 & 0.01279088 & 0.01279088 & 0.01279088 & 0.01279032 & 0.01278973 \\
\hline \multirow{3}{*}{$\operatorname{PRE}\left(\phi, \bar{y}_{\mathrm{AD}}\right)$} & 10 & 0.182 & 100 & 100 & 100 & 100.0516 & 100.1061 \\
& 20 & 0.364 & 100 & 100 & 100 & 100.0205 & 100.0422 \\
& 30 & 0.545 & 100 & 100 & 100 & 100.0097 & 100.0199 \\
& 40 & 0.727 & 100 & 100 & 100 & 100.0044 \\
\hline
\end{tabular}

TABLE 4: MSE and PRE values of different estimators about population IV.

\begin{tabular}{|c|c|c|c|c|c|c|c|}
\hline & $n$ & $n / N$ & $\bar{y}_{\mathrm{AD}}$ & $\bar{y}_{\mathrm{KC}}$ & $\bar{y}_{\mathrm{MS}}$ & $\bar{y}_{\mathrm{RE}}$ & $\bar{y}_{\mathrm{pr}}$ \\
\hline \multirow{4}{*}{ MSE } & 10 & 0.333 & 21.30709 & 21.30709 & 21.30709 & 21.30557 & 17.16434 \\
\hline & 15 & 0.500 & 10.54174 & 10.54174 & 10.54174 & 10.54137 & 9.52763 \\
\hline & 20 & 0.667 & 6.70838 & 6.70838 & 6.70838 & 6.70823 & 6.29770 \\
\hline & 25 & 0.833 & 3.51391 & 3.51391 & 3.51391 & 3.51387 & 3.40123 \\
\hline \multirow{4}{*}{$\operatorname{PRE}\left(\phi, \bar{y}_{\mathrm{AD}}\right)$} & 10 & 0.333 & 100 & 100 & 100 & 100.0071 & 124.1358 \\
\hline & 15 & 0.500 & 100 & 100 & 100 & 100.0035 & 110.6438 \\
\hline & 20 & 0.667 & 100 & 100 & 100 & 100.0022 & 106.5210 \\
\hline & 25 & 0.833 & 100 & 100 & 100 & 100.0012 & 103.3129 \\
\hline
\end{tabular}

fractions. From this viewpoint, the proposed estimator can save survey cost. In some sampling yields, the sample fraction is not very large due to the irreversibility or the high cost of the test. Then the accuracy of the proposed estimator is higher. Haq and Shabbir [15] also reported on estimators of finite population mean using two auxiliary attributes and found that their MSE values were reduced when sample size increased. Therefore, the findings of the present are consistent with that study.

\section{Conclusions}

In this paper, we proposed the improved estimator of a finite population mean by utilizing information on two auxiliary variables in SRS. Bias and MSE expressions of the proposed estimator, $\bar{y}_{\mathrm{pr}}$, were obtained. We clearly proved that the new estimator is always more efficient than the estimators reported by Abu-Dayyeh et al. [7], Kadilar and Cingi [8], and Malik and Singh [9], as well as the regression estimator using two auxiliary variables. These theoretical conditions are also satisfied by the results of four numerical examples in agricultural, biomedical, and power engineering. It should be noted that a smaller sample size yields better results relative to MSE and PRE values when compared to a larger sample size. Thus, for use with small sample size, the suggested estimator would be cost-saving in actual practice and are, therefore, recommended for efficient estimation of finite population mean.

\section{Conflicts of Interest}

The author declares that they have no conflicts of interest.

\section{Acknowledgments}

This work was supported by the National Natural Science Foundation of China (no. 11461051) and Natural Science Foundation of Inner Mongolia Autonomous Region of China (no. 2017MS(LH)0101), and the author gratefully acknowledges this support.

\section{References}

[1] H. P. Singh and G. K. Vishwakarma, "Modified exponential ratio and product estimators for finite population mean in double sampling," Austrian Journal of Statistics, vol. 36, pp. 217-225, 2007.

[2] L. K. Grover and P. Kaur, "An improved estimator of the finite population mean in simple random sampling," Model Assisted Statistics and Applications, vol. 6, no. 1, pp. 47-55, 2011.

[3] L. K. Grover and P. Kaur, "An improved exponential estimator for finite population mean in simple random sampling using an auxiliary attribute," Applied Mathematics and Computation, vol. 218, no. 7, pp. 3093-3099, 2011.

[4] R. Singh, S. Malik, M. K. Chaudhary, H. K. Verma, and A. Adewara, "A general family of ratio-type estimators in systematic sampling," Journal of Reliability and Statistical Studies, vol. 5, pp. 73-82, 2012.

[5] H. P. Singh and R. S. Solanki, "Improved estimation of population mean in simple random sampling using information on auxiliary attribute," Applied Mathematics and Computation, vol. 218, no. 15, pp. 7798-7812, 2012.

[6] S. Gupta and J. Shabbir, "On improvement in estimating the population mean in simple random sampling," Journal of Applied Statistics, vol. 35, no. 5-6, pp. 559-566, 2008.

[7] W. A. Abu-Dayyeh, M. S. Ahmed, R. A. Ahmed, and H. A. Muttlak, "Some estimators of a finite population mean using 
auxiliary information," Applied Mathematics and Computation, vol. 139, no. 2-3, pp. 287-298, 2003.

[8] C. Kadilar and H. Cingi, "A new estimator using two auxiliary variables," Applied Mathematics and Computation, vol. 162, no. 2, pp. 901-908, 2005.

[9] S. Malik and R. Singh, "An improved estimator using two auxiliary attributes," Applied Mathematics and Computation, vol. 219, no. 23, pp. 10983-10986, 2013.

[10] P. Sharma and R. Singh, "A class of exponential ratio estimators of finite population mean using two auxiliary variables," Pakistan Journal of Statistics and Operation Research, vol. 11, no. 2, pp. 221-229, 2015.

[11] S. Muneer, J. Shabbir, and A. Khalil, "Estimation of finite population mean in simple random sampling and stratified random sampling using two auxiliary variables," Communications in Statistics. Theory and Methods, vol. 46, no. 5, pp. 2181-2192, 2017.

[12] T. J. Rao, "On certain methods of improving ratio and regression estimators," Communications in Statistics. Theory and Methods, vol. 20, no. 10, pp. 3325-3340, 1991.

[13] H. P. Singh and M. R. Espejo, "On linear regression and ratioproduct estimation of a finite population mean," Journal of the Royal Statistical Society. Series D. The Statistician, vol. 52, no. 1, pp. 59-67, 2003.

[14] S. Choudhury and B. K. Singh, "A class of chain ratio-product type estimators with two auxiliary variables under double sampling scheme," Journal of the Korean Statistical Society, vol. 41, no. 2, pp. 247-256, 2012.

[15] A. Haq and J. Shabbir, "An improved estimator of finite population mean when using two auxiliary attributes," Applied Mathematics and Computation, vol. 241, pp. 14-24, 2014.

[16] H. K. Verma, P. Sharma, and R. Singh, "Some families of estimators using two auxiliary variables in stratified random sampling," Investigacion Operacional, vol. 36, no. 2, pp. 140-150, 2015. 


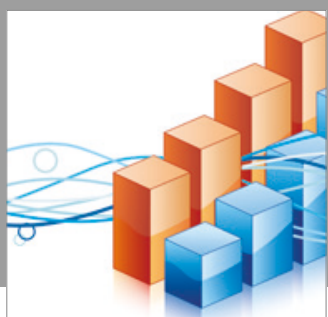

Advances in

Operations Research

vatersals

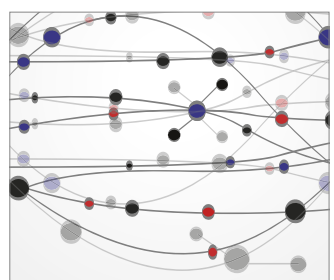

\section{The Scientific} World Journal
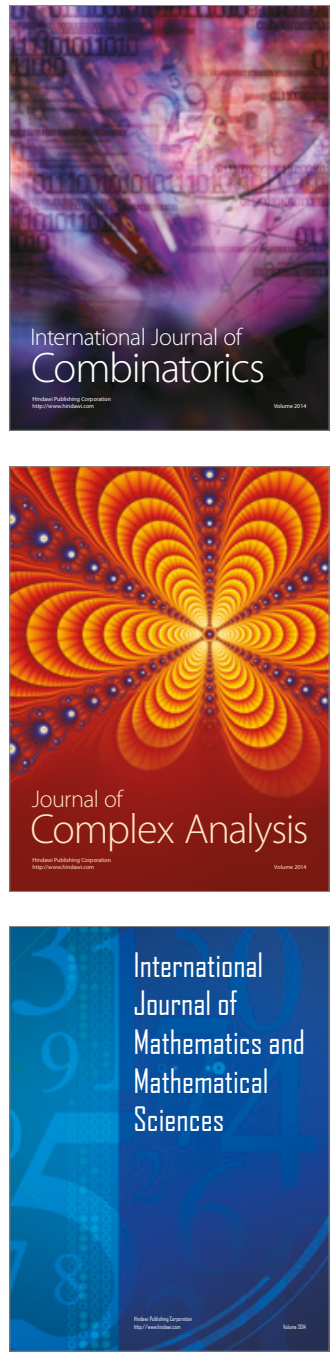
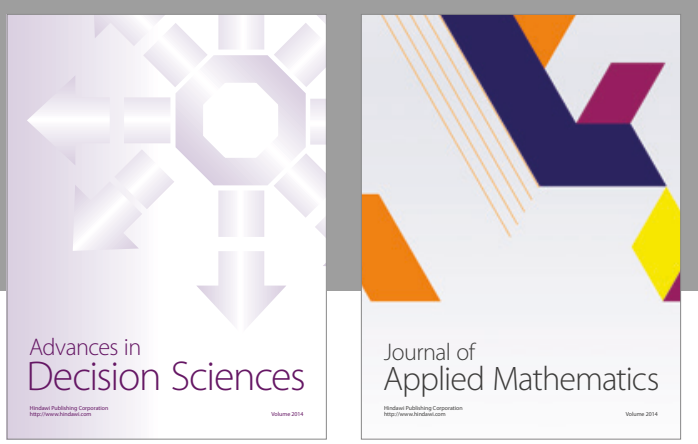

Algebra

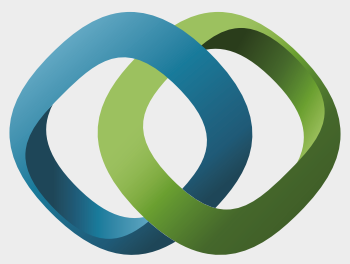

\section{Hindawi}

Submit your manuscripts at

https://www.hindawi.com
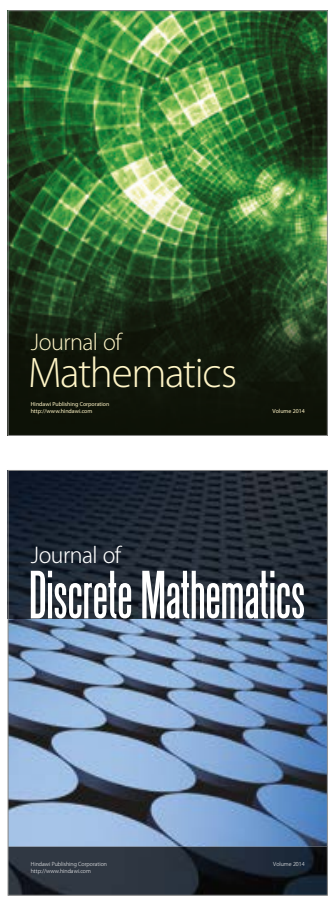

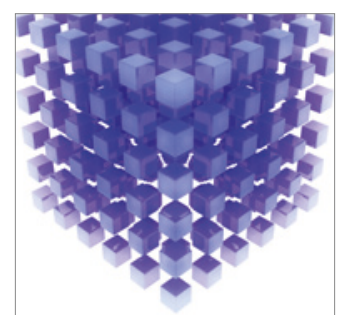

Mathematical Problems in Engineering
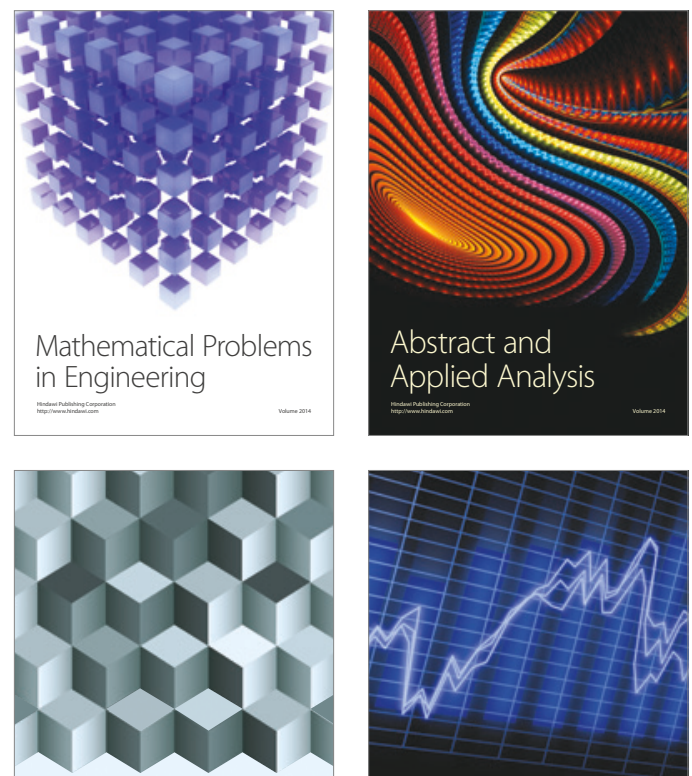

Journal of

Function Spaces

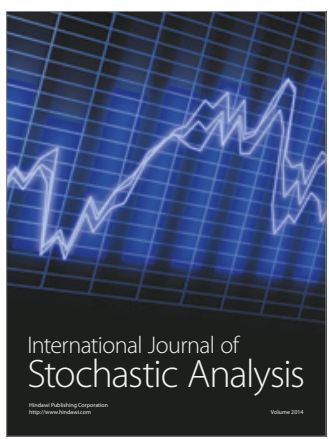

Probability and Statistics
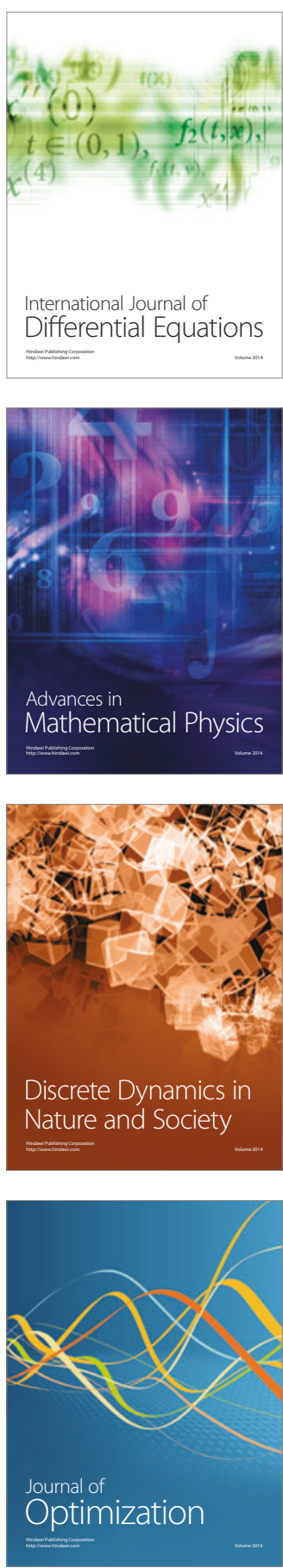